\title{
Evaluation of fog warning system on driving under heavy fog condition based on driving simulator
}

\author{
Xiaohua Zhao and Xuervei Li \\ Beijing University of Technology, Beijing, China and Tsinghua University State Key Laboratory of Automotive Safety and Energy, \\ Beijing, China \\ Yufei Chen \\ China Automotive Engineering Research Institute, Beijing, China, and \\ Haijian Li and Yang Ding \\ Beijing University of Technology, Beijing, China
}

\begin{abstract}
Purpose - Heavy fog results in low visibility, which increases the probability and severity of traffic crashes, and fog warning system is conducive to the reduction of crashes by conveying warning messages to drivers. This paper aims at exploring the effects of dynamic message sign (DMS) of fog warning system on driver performance.

Design/methodology/approach - First, a testing platform was established based on driving simulator and driver performance data under DMS were collected. The experiment route was consisted of three different zones (i.e. warning zone, transition zone and heavy fog zone), and mean speed, mean acceleration, mean jerk in the whole zone, ending speed in the warning zone and transition zone, maximum deceleration rate and mean speed reduction proportion in the transition zone and heavy fog zone were selected. Next, the one-way analysis of variance was applied to test the significant difference between the metrics. Besides, drivers' subjective perception was also considered.

Findings - The results indicated that DMS is beneficial to reduce speed before drivers enter the heavy fog zone. Besides, when drivers enter a heavy fog zone, DMS can reduce the tension of drivers and make drivers operate more smoothly.

Originality/value - This paper provides a comprehensive approach for evaluating the effectiveness of the warning system in adverse conditions based on the driving simulation test platform. The method can be extended to the evaluation of vehicle-to-infrastructure technology in other special scenarios.
\end{abstract}

Keywords Heavy fog conditions, Fog warning system, Dynamic message sign, Driver performance, Driving simulator

Paper type Research paper

\section{Introduction}

Adverse weather conditions might cause many traffic crashes on the freeway. The US Department of Transportation has analyzed the traffic crashes in detail. It shows that crashes caused by adverse weather conditions account for $23 \%$ of the road traffic crashes. The amount of fog crashes accounts for $3 \%$, while the death toll of fog crashes accounts for $9 \%$. In all of the traffic crashes on the freeway in foggy weather: traffic crashes involving just one car account for $18.75 \%$, crashes involving two cars account for $23.9 \%$, crashes involving more than two cars account for $57.29 \%$, crashes without casualties only account for $2 \%$, crashes involving one to three deaths account for $38 \%$, crashes involving ten or more deaths account for $11 \%$ (Zhong, 2008 and Zhang, 2009). Because of the low visibility of the heavy fog, the traffic crashes might be more frequent and serious.

The current issue and full text archive of this journal is available on Emerald Insight at: https://www.emerald.com/insight/2399-9802.htm

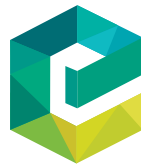

Journal of Intelligent and Connected Vehicles $4 / 2(2021) 41-51$

Emerald Publishing Limited [ISSN 2399-9802] [DOI 10.1108/JICV-11-2020-0012]
Essentially, the core reason why there are many traffic crashes in foggy weather is the negative impact of the reduced visibility on driving behavior. At present, among the different driving behavior indexes, speed and acceleration are most obviously affected by fog. Hawkins (1988) found that when the visibility was low but higher than $150 \mathrm{~m}$ in fog, the speed of the driver was higher than that in sunny weather. When the visibility continued to drop to $100 \mathrm{~m}$, the speed of the driver decreased significantly. Snowden et al. (1998) discovered that simulated scenarios appeared to move more slowly in foggy

(C) Xiaohua Zhao, Xuewei Li, Yufei Chen, Haijian Li and Yang Ding. Published in Fournal of Intelligent and Connected Vehicles. Published by Emerald Publishing Limited. This article is published under the Creative Commons Attribution (CC BY 4.0) licence. Anyone may reproduce, distribute, translate and create derivative works of this article (for both commercial and non-commercial purposes), subject to full attribution to the original publication and authors. The full terms of this licence maybe seen at http://creativecommons.org/licences/by/4.0/legalcode

This research is sponsored by the State Key Laboratory of Automotive Safety and Energy under Project No. KF2017.

Received 4 November 2020

Revised 19 January 2021

4 March 2021

Accepted 12 March 2021 
conditions than in warning conditions, and participants drove faster in fog days to achieve a "target speed." Hoogendoorn (2010) studied the driving behavior with a driving simulator, and the results showed that fog caused the decrease of speed and acceleration, and the distance from the vehicle in front increased during the following process. Brooks (2011) pointed out that the driver would continue to maintain a high driving speed in light fog, while Brooks' research results show that when the visibility drops to about $150 \mathrm{~m}$, the driver will gradually reduce the driving speed of the vehicle. Although the driver reduces the speed in foggy weather, Abdel (2011) indicated that the speed reduced by the driver in foggy weather cannot guarantee driving safety. It is revealed that drivers would like to reduce their speed to lower the risk in fog conditions (Wu, 2018). In addition, Chen (2010) found that about $70 \%$ of the drivers have excessive tension when entering the fog zone. From the above studies, it can be concluded that visibility can cause drivers to change the speed and acceleration because the biggest problem in foggy weather is the declined visibility which might lead to the untimely response to emergencies and produce psychological tension. Moreover, the change is more obvious when the visibility less than $150 \mathrm{~m}$. If the driver can be informed of the existence of fog zone before reaching the fog zone, the emergency response and the level of psychological tension of the driver can be effectively reduced. At present, the fog warning system provides a better way to solve the emergency response of drivers to fog zone. The presence of fog is detected by installing visibility sensor or camera on the roadside, and the fog information was transmitted to the management center and then the management center notifies drivers by broadcasting or displaying on the sign.

Nowadays, the fog warning system is widely used to improve the driving safety of highway in foggy days. Among them, dynamic message sign (DMS) is an effective means to implement fog warning system. As a kind of intelligent roadside terminal in intelligent vehicle road cooperation system, DMS can communicate with the management system and plays an important role in adverse weather and traffic emergency warning. Especially in the case of highway fog warning, it can timely and effectively convey the weather information of the road ahead and reasonably guide the driver to help to guarantee the traffic safety. DMS is an infrastructure for real-time display of information sent by management center and is usually located in front of the fog zone, which helps the drivers adjust the driver performance when they enter the fog zone. In the USA, UT (Goodwin, 2013), TN (Pilié, 1975), WA (Pisano, 2004), Carolina (Xu, 2007) have the fog warning systems, where DMS and speed limit signs are installed on the roadside to reduce the speed of vehicles under adverse weather conditions to reduce the accident rates. In Japan, fog warning system includes speed limit sign, traffic control device and DMS. A research shows that the effect of fog warning system is remarkable (Xu, 2007). In Australia, DMS is used to carry out the warning measures in foggy days on the freeway. Moreover, the reduction of vehicle speed and the difference of speed after the implementation of the measures is analyzed, which verifies the effectiveness of the facilities ( $\mathrm{Xu}, 2007)$. Germany has set up weather detection equipment along the road because the beginning of the decade to detect the impact of climate change on road with the number of weather stations increasing since then. The detected information is aggregated to the German national meteorological service center, which publishes weather information and studies new weather forecasting methods ( $\mathrm{Xu}, 2007)$. Although China attaches great importance to the management of fog warning, the widespread application of fog warning system needs to be further consolidated because of the unsystematic detecting equipment and there is no established norm for fog warning.

Additionally, the effectiveness of the equipment is also of great importance, mainly for the interaction between the release of DMS and drivers. Many scholars have studied the effects of fog warning systems to evaluate the impact of fog warning systems on drivers' decisions. Boyle and Mannering analyzed the driver's speed adjustment under driving simulation conditions for four different advisory messages. The results of this research showed that warning information is effective on the speed of the fog zone. While driving in the fog zone, the drivers usually increased the speed at downstream of the fog zone (Boyle, 2004). Al-Ghamid et al. (2007) have shown that the fog warning system can reduce the average speed of the driver in the fog zone significantly, but the speed change is not desirable. Hassan and Abdel-Aty (2011) used subjective questionnaires to research drivers' trust and obedience factors. The research showed that drivers are more compliant with fog warning systems in heavy fog and fog conditions. Wu et al. (2018) studied the effects of different fog warning systems on drivers' speed. The results showed that the fog warning system is effective in reducing speed. In conclusion, the fog warning system can forecast the status of fog zone for drivers in advance. Therefore, fog warning system can effectively reduce the driving speed and improve safety in the fog zone. However, there is still much room for improvement in the real-time and the accuracy of the system, and the fog warning system needs to be further evaluated.

There are two methods of the evaluation of fog warning system: driving simulator test (Zhao, 2018) and field test (Wang, 2016). Heavy traffic crashes are easy to occur under heavy fog (visibility $=125 \mathrm{~m}$ ). The field test has the advantages of real environment and real data, but in field test, the real-car test cannot be carried out because heavy fog is not common; therefore, the real-car test has certain limitations. Nevertheless, a driving simulator has multiple advantages over dangerous conditions, unusual weather:

- flexible experiment conditions;

- the ability to provide crash-prone scenarios that are often unsafe to perform in real-life situations;

- economical;

- less time needed to expose participants to a wide variety of situations; and

- convenient to objectively measure driver performance (Godley et al., 2002; Zhao et al., 2019).

In addition, the driving simulator can collect refined data to assess the changes of driving behavior, and its effectiveness when applied in real-world scenarios has been confirmed in previous studies (Charlton, 2009).

In this research, a fog warning system effective testing platform based on driving simulator is established, and the effect of the fog warning system on driver performance adjustments under heavy fog conditions was quantified. The fog warning system in this 
research is different from those in previous studies. It can provide the driver with not only information about the concentration of fog but also the distance from the fog zone. The research includes three parts: Part 1 introduces the structure of fog warning system and testing platform based on driving simulator. In Part 2, the driving simulator experiment is instructed. Part 3 evaluates the drivers' performance on DMS in foggy environment based on the data of simulation experiment and subjective questionnaires. This paper proposes an improved fog warning system on account of the previous research and actual road management measures and forms a comprehensive approach for fog warning system test by a dynamic interactive simulation test platform. The current research provides a method to explore the impact of VICS information (provided by vehicle terminal and intelligent road terminal on driving performance and helps to improve the safety performance of vehicle infrastructure cooperative system and to deploy the control strategy of connected vehicle system in special scenarios.

\section{Platform design and development}

\subsection{Fog warning system}

The fog warning system proposed in this paper is an improvement of previous studies to some extent. First, the basic form is determined by referring to the paper (Wu, 2018); second, based on the visibility levels (GBT 27964-2011) and the standard of Monitoring of Visibility and Warning of Heavy Fog on Highway (QX/T76-2007), the fog level and distance are added to increase the driver's situation awareness and prediction ability. Moreover, considering the convenience of observation and the actual freeway facilities settings, the roadside DMS is adjusted to doorframe type DMS; Finally, according to the calculation method of DMS, combined with the suggestions on preliminary notice traffic signs in national standard (GB 51038-2015), the multi-level warning was carried out in this paper. The specific introduction of the fog warning system is as follows.

\subsubsection{Basic composition of fog warning system}

In reality, the fog warning system consists of DMS, visibility sensor, distance sensor and management center and is usually set in the fog-prone zone, whose framework is as follows:

- Data collection: Visibility sensor and distance sensor are installed in the fog-prone zone. The visibility sensor is installed each $400 \mathrm{~m}(\mathrm{Xu}, 2007)$ and the distance sensor is installed each $100 \mathrm{~m}$, the distance between the DMS and the fog zone can be expressed by the distance between the sensors.

- Visibility and distance perception: When the visibility is less than $10,000 \mathrm{~m}$, the information will be passed to the management center. The management center compares the visibility information with the threshold value of the classification standard related to fog level to determine the level of fog (Table 1). Meanwhile, the distance sensor at the beginning of fog zone communicates with the DMS to confirm the distance that is shown on the DMS from fog zone.

- Warning execution: The management center displays the distance and fog level information in the DMS. For example, "Heavy fog one kilometer ahead, please be careful" is showed in the DMS which is set in the zone that is $1 \mathrm{~km}$ in front of the fog zone (Figure 1). The drivers can receive the warning information when they see the DMS.
Table 1 Visibility levels in China (GBT 27964-2011)

\begin{tabular}{ll}
\hline Visibility & Level \\
\hline $1,000 m \leq V<10000 m$ & Light fog \\
$500 m \leq V<1000 m$ & Fog \\
$200 m \leq V<500 m$ & Strong fog \\
$50 m \leq V<200 m$ & Heavy fog \\
$V<50 m$ & Strong heavy fog \\
\hline
\end{tabular}

Figure 1 Design of DMS

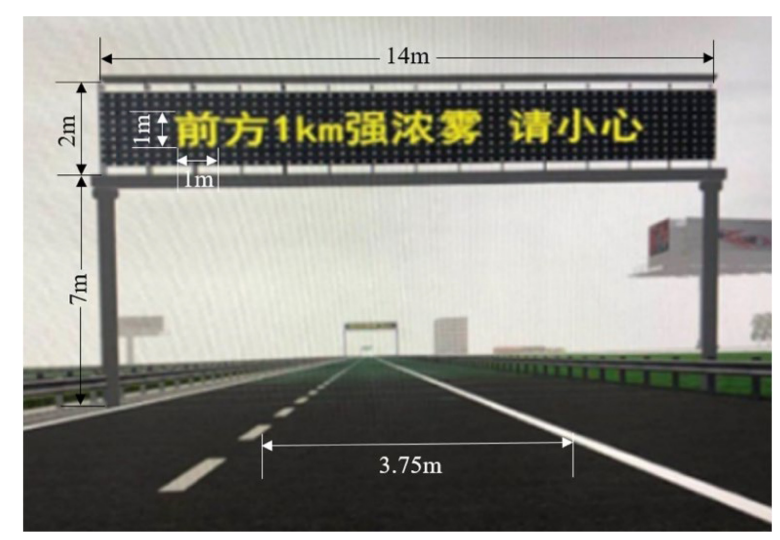

\subsubsection{Dynamic message sign design}

2.1.2.1 Color of dynamic message sign. The DMS plays an important role of warning the driver. In China, the color of DMS's layout text has different meanings: red means ban, yellow means warning and green means permission. Therefore, yellow is chosen as the color of DMS text in this system.

2.1.2.2 Setting mode of dynamic message sign. Traffic signs are set up in columns type (both single and double), cantilevered type, attached type and doorframe type (JTG D82-2009). The DMS on the freeway adopts the doorframe type through communicating with traffic management department because the doorframe sign is more striking than the roadside sign. The size of the DMS is shown in Figure 1 (take two lanes, for example, the lane width is $3.75 \mathrm{~m}$ ).

2.1.2.3 Advance distance of dynamic message sign. To enhance the effect of forecast, multiple DMS should be set up. The advance distance of DMS should be considered to ensure that the driver can see each DMS. DMS theoretical advance distance can be calculated (Formula 1) (Wang, 2009). According to the formulation, in theory, the advance distance of DMS is $371 \mathrm{~m}$, which means that the driver can see all the DMS when the actual advance distance of DMS is greater than $371 \mathrm{~m}$. Therefore, the advance distance and spacing of fog zone of DMS in this research are $500 \mathrm{~m}$ :

$$
D \geq(n-1) \frac{V_{1}}{3.6} \times t_{2}+\frac{V_{1}^{2}-V_{2}^{2}}{254(f+i+\varphi)}+\frac{V_{1}}{3.6} \times t_{1}-(b+4.3) / \tan a
$$

where $\mathrm{D}$ is vanishing point of DMS. $\mathrm{N}$ is the number of twoway lanes, take 4 (four lanes in two directions). $V_{1}$ is the speed $(\mathrm{km} / \mathrm{h})$ when reading, $85 \%$ running speed or limited speed can 
be adopted, take $102 \mathrm{~km} / \mathrm{h} . V_{2}$ is the speed after taking action, the minimum speed can be adopted, which is $60 \mathrm{~km} / \mathrm{h}$. $f$ is rolling resistance coefficient; asphalt concrete pavement is $0.015 . i$ is the total slope of the road, usually take $3 \% . \varphi$ is adhesion coefficient of pavement, 0.6 for asphalt concrete pavement. $a$ is the vanishing point and the top edge of roadside sign marks the elevation, usually take $15^{\circ} . b$ is DMS height, take $10 \mathrm{~m} . t_{1}$ is total time of perception and braking reaction, usually take $2.5 \mathrm{~s}$. $t_{2}$ is the time required for a vehicle to change lanes once, usually take $6.5 \mathrm{~s}$.

\subsection{Testing platform design}

The research constructed a fog warning system test platform based on driving simulation to evaluate its effectiveness. The fixed-base driving simulator located in the Key Laboratory of Traffic Engineering of Beijing University of Technology consists of a real car, computers, videos and audio equipment. The road scenario was projected onto three big screens, providing a 130-degree field of view. The screen resolution of the driving simulator was $1,920 \times 1,080$. The simulator recorded operating data (e.g. braking force, acceleration, speed, lateral placement, lane numbers and turning angle of the steering wheel) 30 times per s. The simulator adopted an Application Programming Interface, which allowed users to design driving tasks according to their needs, such as weather, signs and other elements.

We used the driving simulator to develop a 3D scene landscape to create the fog environment, and we simulated and recreated the components of the fog warning system. First, the Application Programming Interface (API) collected the weather and distance data in the scene from the Driving Simulator System (DSS), the interconnection was structured between driving simulator and DSS through interface, which corresponded to the data collection by sensor in the actual system. The virtual visibility sensor and distance sensor collected data. DSS and management center synchronously transmitted through the User Datagram Protocol (UDP) corresponding and transmitted the distance and visibility information that were collected in the scene to the management center, which corresponded to the visibility and distance perception in the actual system. Management center sent the final display information to DMS by comparing with the threshold value and the warning information received by drivers through the DMS, corresponding to warning execution in the actual system. If the sensor detected that there is a change in data, the process would be roll-back (Figure 2).

The establishment of the test platform of fog warning system has certain significance for the effectiveness evaluation of fog warning

Figure 2 Driving simulation platform

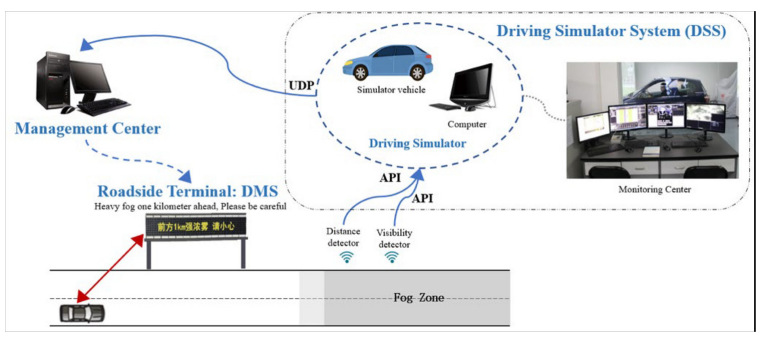

system before implementation. The test platform provides a test basis for the warning system, and a set of evaluation method of warning system is formed, which can be extended to similar warning system, such as the work zone tunnel and long downhill warning systems (Zhao et al., 2019). Based on the established test platform, we obtained abundant driving behavior data to evaluate the fog warning system that we designed.

\section{Method}

To evaluate the effectiveness of fog warning system, a driving simulation experiment was designed.

\subsection{Participants}

A total of 43 healthy participants (age: $M=35$ years, $S D=$ 11.88), including 28 males and 15 females, were recruited from universities and social organizations. The participants were required to have normal or corrected vision (self-reported, participants were required to wear an eye tracker) and no hearing problems (self-reported). All participants were provided with written informed consent before joining the experiment. Drivers could not participate in the training until providing informed consent. After complementing the driving task, each participant was offered China Yuan (CNY) 200 cash as a reward.

The selection of sample size is the key to obtain sufficient experimental data. A sample size which is too small may reduce the credibility of results, while too much, the sample size will lead to the waste of resources and time. For example, the research that studied the influence of roadside infrastructure on vehicle operation was conducted with 22 participants (Van, 2018); moreover, the research that studied driving behavior in foggy weather based on driving simulation with 19 participants (Hoogendoorn, 2010). This research calculated the required sample size using equation (2):

$$
n=\frac{\left(z_{\alpha / 2}+z_{\beta}\right)^{2} \sigma^{2}}{\varepsilon^{2}}
$$

where $z_{\alpha / 2}$ is the upper $(\alpha / 2)$ th quantile of the standard normal distribution; $z_{\beta}$ is the upper $(\beta)$ th quantile of the standard normal distribution; $\sigma$ is the standard deviation of the normal distribution population; $\varepsilon$ is the difference between the true mean response of a test factor and a reference value, which can be given by $\varepsilon= \pm \delta \sigma$ (Lerman, 1996), $\delta$ is the meaningful difference, in practice, a value between 0.25 and 0.5 is usually chosen as $\delta$ if no previous knowledge (Chow et al., 2017). Typically, a $10 \%$ level of significance is chosen to reflect a $90 \%$ confidence regarding the unknown parameter. To balance the power and cost-effectiveness, a power of $80 \%$ and a meaningful difference of 0.5 were used. The results showed that the required sample size in this research was 25 . This implied that the experiment design could provide reliable answers to the questions to be investigated. Therefore, the sample size of this research is in line with the requirement Table 2.

\subsection{Scenario design}

Xingyan freeway connects Beijing and Yanqing in China and will expand to Chongli where the 2022 winter Olympics will be hosted. The research designed the experimental scenario based on linear section of Xingyan freeway with a total length of $6,060 \mathrm{~m}$. All scenarios were set on a two-way, four-lane freeway 
Table 2 Demographics summary

\begin{tabular}{|c|c|c|}
\hline \multirow[b]{2}{*}{ Demographic variables } & \multicolumn{2}{|c|}{$\begin{array}{l}\text { Mean (SD) statistics } \\
\text { or percentages }\end{array}$} \\
\hline & $\begin{array}{c}\text { Male } \\
\text { participants }\end{array}$ & $\begin{array}{c}\text { Female } \\
\text { participants }\end{array}$ \\
\hline Age (years) & $37.5(13.1)$ & $25(12.97)$ \\
\hline Age of license (years) & $16(10.2)$ & $13(9.3)$ \\
\hline $\begin{array}{l}\text { Average driving mileage } \\
\text { (per year/km) }\end{array}$ & $18,524(3548.22)$ & $9,584(5514.21)$ \\
\hline
\end{tabular}

modeled on the Xingyan freeway with a $22.4 \mathrm{~m}$ cross-section (lane width $=3.75 \mathrm{~m}$, median (green belt) width $=2.6 \mathrm{~m}$ and shoulder width $=4.55 \mathrm{~m}$ ) and a speed limit of $60-120 \mathrm{~km} / \mathrm{h}$ in normal road. The experiment selected the road to serve the winter Olympics.

This study is a part of a large-scale experiment. The whole experiment included three factors: intelligent roadside facilities (DMS on; DMS off), intelligent vehicle terminal (humanmachine interface: HMI on; HMI off) and fog level (no fog, light fog, heavy fog), respectively. At the same time, the experimental sequence was randomly arranged, and the driver did not know what kind of weather or facilities he will encounter before each trail, which reduced the impact of the driver's expectation of the experimental environment on driving performance.

In this research, only heavy fog (visibility $=125 \mathrm{~m}$ ) was selected as the experimental fog zone, and free flow was selected as the traffic flow. There was one factor and two levels in this study: without DMS (treat group) [Figure 3(a)] and with DMS (control group) [Figure 3(b)]. Treat group had a total of four zones: speed up zone, warning zone, transition zone and heavy fog zone. The speed up zone was $1,500 \mathrm{~m}$, which was set for the driver to speed up to a stable speed. The total warning zone was $1,800 \mathrm{~m}$. The visibility of the warning zone was greater than 1,000 m (GBT 27964-2011). The total transition

\section{Figure 3 Scenario design}

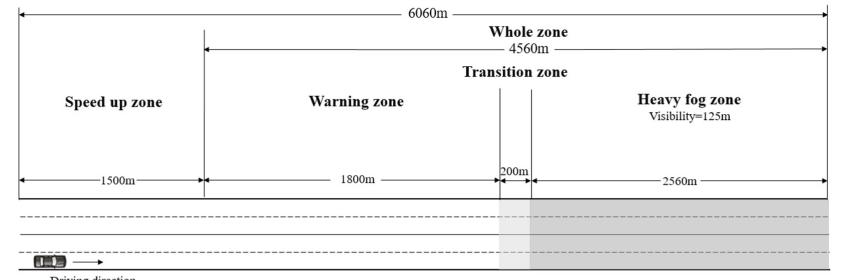

(a)

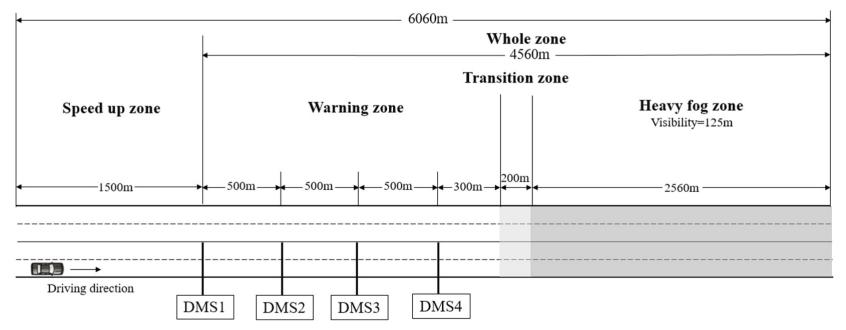

(b)

Notes: (a) Treat group: Without DMS; (b) control group: With DMS zone was $200 \mathrm{~m}$, the visibility of which would gradually decreased to make the driver adapted to heavy fog. Heavy fog zone was $2,560 \mathrm{~m}$ and the visibility was $125 \mathrm{~m}$, which can stabilize driver's speed. The control group was the same as the treat group except that there are four DMS included in the warning zone, the function of the warning zone was to provide the distance and concentration of fog for drivers in advance. According to the system design, the spacing between each DMS was $500 \mathrm{~m}$. Experimental data analysis was applied to the warning zone, transition zone and heavy fog zone on account of the unstable data in the speed zone. We call the sum of warning zone, transition zone and heavy fog zone as the whole zone, which is used to get a clearer change of the driving performance in the whole area Figure 4.

\subsection{Experiment procedure}

First, all participants were given the following instructions before driving on the simulator:

You are about to participate in a driving experiment on the simulator. During the process of driving, please drive the way as you normally do, but obey the traffic laws, the speed limit of the freeway is $120 \mathrm{~km} / \mathrm{h}$.

Second, participants were investigated through questionnaires for basic information and the degree of fatigue. Their initial responses were logged as a baseline for comparison throughout the experiment to check for any adverse reactions to the simulator. Third, after the questionnaires, participants performed pre-driving practice for approximately 3-5 min. The main purpose of this practice was to minimize the impact of participants' unfamiliarity with the driving simulator and to check if the driver has motion sickness. Fourth, the formal experiment was conducted. Each experiment last about $8 \mathrm{~min}$, with at least $5 \mathrm{~min}$ of rest between experiments. At last, after the driving experiment, each driver filled out the after-driving questionnaire to acquire the degree of fatigue after driving and the subjective performances of the experiment.

Figure 4 Display of DMS

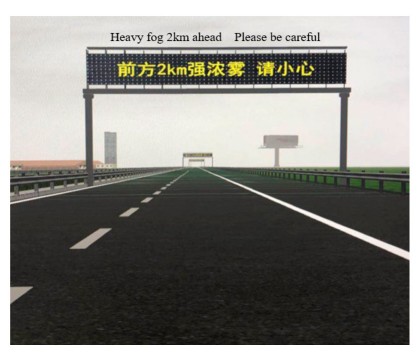

(a)

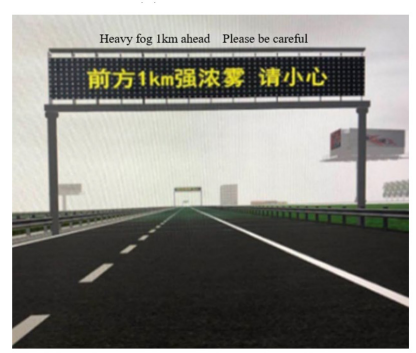

(c)

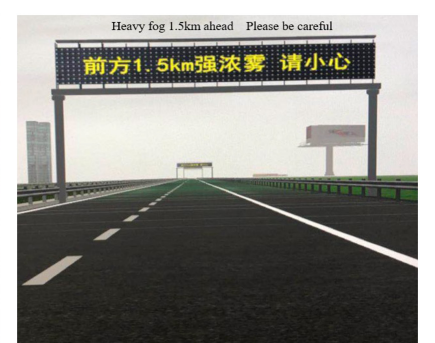

(b)

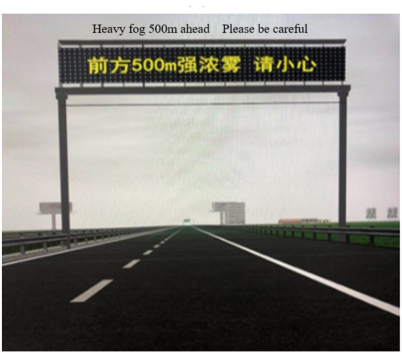

(d)
Notes: (a) DMS1; (b) DMS2; (a) DMS3; (b) DMS4 


\section{Results}

The experiment obtained the experimental data of 43 drivers. However, two drivers developed motion sickness and three drivers were speeding. Therefore, a total of 38 drivers' valid data were analyzed. The research analyzed the fog warning system from subjective and objective aspects. In subjective aspect, drivers' subjective feelings about the fog warning system were evaluated based on the subjective questionnaire including drivers' acceptance, understanding, trust of the DMS. In objective aspect, the indicators of drivers' performance were mainly used to reflect the effectiveness of DMS for drivers. In the research, one-way ANOVA model was applied for data analysis for the scenario with DMS and the scenario without DMS. To better get the impact section in detail and performed spatial significance assessment, the data was intercepted every $20 \mathrm{~m}$ by MATLAB. In addition, data significance analysis was conducted from two dimensions of time and space, represented by a step diagram, 1 represents a significant difference between two points, and 0 represents no significant difference between two points. For all experiments, a $p$-value $<0.05$ is considered significant.

Considering the consistency of the entering speed of each region so as to assess the consistency of the effect, DMS3 and DMS4 were taken as the main research objects. Other studies have also mentioned that the function of two DMS should be considered in settings (Wu, 2018).

\subsection{Subjective questionnaire analysis}

To evaluate drivers' subjective understanding and trust in the DMS of the fog warning system, a subjective questionnaire was designed and was conducted (Table 3 ) after driving.

The reliability and validity of the questionnaire were tested. The test results showed that the Cronbach's alpha $(\alpha)$ for all the scales is 0.613 and the Kaiser-Meyer-Olkin value is $0.680, P=0.000$, which shows that the questionnaire results are effective and reliable.

In the process of driving, most of the drivers considered that it is reasonable to set the DMS (92.31\%) and agreed that the prompt can improve the driving safety $(82.05 \%)$. DMS should be set up in front of the heavy fog zone $(92.3 \%)$. Most drivers considered that the DMS do not have negative impact on the process of driving (79.49\%). Because the DMS is the warning sign, most drivers $(71.79 \%)$ chose normal driving, while a few drivers $(28.21 \%)$ selected to gradually reduce the speed. The Pearson correlation analysis shows that drivers who considered that the DMS is reasonable also agreed that it can improve safety in fog zone (Pearson correlation $=0.499$ ). Drivers who considered that the fog warning system can improve safety generally agreed that the DMS should be set ahead of the fog zone (Pearson correlation $=0.414)$. In conclusion, drivers had a subjective attitude of trust and support for the DMS of the fog warning system and can correctly understand the meaning of the DMS.

\subsection{Indicators of driver performance}

Normally, there are four performance criteria that can analyze the driving behavior in terms of benefits and costs potential: safety, an opportunity for action, driver comfort and time spent (Vanderhaegen, 2011). The experiment road was divided into three parts: warning zone, transition zone and heavy fog zone. The safety indicator of the driver was represented by the mean speed $\left(V_{\text {mean }}\right)$ in the whole zone. The comfort indicator of the driver was represented by the mean acceleration $\left(a_{\text {mean }}\right)$ and

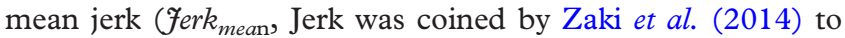
describe the driver behavior in expressway. Jerk is the derivative of acceleration to time, reflecting the speed of acceleration change and representing the driver's adaptation to acceleration change. The larger the value indicates the more drastic the change of the driving state, the more unstable the physiological state of the driver and the worse the comfort) in whole zone.

To distinguish different regions, the corresponding letters were added to the indicators of each region ( $W$ represents warning zone, $T$ represents transition zone, $F$ represents heavy fog zone). All indicators are shown in Table 4. A significance analysis on all the indicators was performed, and all the significant indicators are shown in Figure 5. $V_{W \text {-end }}$ and $V_{T \text {-end }}$ represent ending speeds in the zone. $a_{T-\max }$ and $a_{F-\max }$ represent the biggest deceleration in the zone. Proportion $_{T-W}$ (Formula 3), Proportion $_{F-W}$ (Formula 4) and Proportion P $-T_{\text {(Formula 5) }}$ represent reduction ratio between the two zones. The unique indicators were chosen by considering the principles of using the least amount of data to represent the driving behavior (Abdel-Aty, 2010). The indicators selection of this paper refers to the research of Yina $\mathrm{Wu}(2018)$ :

Table 3 Design and results of subjective questionnaire

\begin{tabular}{|c|c|c|c|c|c|}
\hline \multirow{2}{*}{$\begin{array}{l}\text { Question } \\
\text { You consider that the DMS setting } \\
\text { and the words are reasonable } \\
\text { during driving }\end{array}$} & \multicolumn{5}{|c|}{ Options and proportions } \\
\hline & $\begin{array}{l}\text { Strongly Agree: } \\
48.72 \%\end{array}$ & $\begin{array}{l}\text { Agree: } \\
43.59 \%\end{array}$ & $\begin{array}{l}\text { Neutral: } \\
7.69 \%\end{array}$ & $\begin{array}{l}\text { Disagree: } \\
0 \%\end{array}$ & $\begin{array}{l}\text { Strongly disagree: } \\
0 \%\end{array}$ \\
\hline The DMS can improve the driving & Strongly Agree: & Agree: & Neutral: & Disagree: & Strongly disagree: \\
\hline safety in the fog zone & $46.15 \%$ & $35.9 \%$ & $15.38 \%$ & $2.57 \%$ & $0 \%$ \\
\hline You consider that the DMS should & Strongly Agree: & Agree: & Neutral: & Disagree: & Strongly disagree: \\
\hline be set before heavy fog zone & $46.15 \%$ & $46.15 \%$ & $7.70 \%$ & $0 \%$ & $0 \%$ \\
\hline The DMS can negatively interfere & Strongly Agree: & Agree: & Neutral: & Disagree: & strongly disagree: \\
\hline with your driving process & $5.13 \%$ & $2.56 \%$ & $12.82 \%$ & $28.21 \%$ & $51.28 \%$ \\
\hline What will you do if you see the & Urgent to slowdown: & Reduce speed slowly: & Normal: & Increase & Urgent to accelerate: \\
\hline DMS while driving & $0 \%$ & $28.21 \%$ & $71.79 \%$ & $\begin{array}{l}\text { speed slowly: } \\
0 \%\end{array}$ & $0 \%$ \\
\hline
\end{tabular}


Table 4 Descriptive statistics of dependent variable

\begin{tabular}{|c|c|c|c|c|c|c|c|c|}
\hline \multirow[b]{2}{*}{ Zone } & \multirow[b]{2}{*}{ Indicator } & \multicolumn{2}{|c|}{ Control group } & \multicolumn{2}{|c|}{ Treat group } & \multirow[b]{2}{*}{$\left(\frac{M_{\text {control }}-M_{\text {treat }}}{M_{\text {control }}}\right)$} & \multirow[b]{2}{*}{ F. } & \multirow[b]{2}{*}{$\begin{array}{c}\text { Mean } \\
p \text {-value }\end{array}$} \\
\hline & & Mean & SD & Mean & SD & & & \\
\hline \multirow[t]{4}{*}{ Warning zone } & $V_{W \text {-mean }}(\mathrm{km} / \mathrm{h})$ & 109.438 & 11.640 & 115.282 & 6.389 & -0.053 & 6.200 & $0.015^{*}$ \\
\hline & $a_{W \text {-mean }}\left(\mathrm{m} / \mathrm{s}^{2}\right)$ & -0.025 & 0.078 & 0.029 & 0.072 & 2.160 & 8.182 & $0.006^{* *}$ \\
\hline & $V_{W \text {-end }}(\mathrm{km} / \mathrm{h})$ & 106.923 & 13.813 & 115.368 & 6.850 & -0.079 & 9.599 & $0.003^{* *}$ \\
\hline & $\operatorname{Jerk}_{W \text {-mean }}\left(\mathrm{m} / \mathrm{s}^{3}\right)$ & -0.0036 & 0.0087 & -0.0078 & 0.0091 & -1.6667 & 3.705 & 0.059 \\
\hline \multirow[t]{6}{*}{ Transition zone } & $V_{T \text {-mean }}(\mathrm{km} / \mathrm{h})$ & 105.365 & 15.870 & 114.463 & 7.774 & -0.086 & 8.480 & $0.005^{* *}$ \\
\hline & $a_{T \text {-mean }}\left(\mathrm{m} / \mathrm{s}^{2}\right)$ & -0.131 & 0.212 & -0.150 & 0.376 & -0.145 & 0.057 & 0.812 \\
\hline & $a_{T-\max }\left(\mathrm{m} / \mathrm{s}^{2}\right)$ & -0.397 & 0.529 & -0.557 & 1.391 & 0.999 & 0.367 & 0.547 \\
\hline & $V_{T \text {-end }}(\mathrm{km} / \mathrm{h})$ & 103.092 & 19.112 & 111.85 & 13.640 & -0.085 & 4.453 & $0.039^{*}$ \\
\hline & Proportion $_{T-W}$ & 0.040 & 0.087 & 0.007 & 0.049 & 0.825 & 3.496 & 0.066 \\
\hline & $\operatorname{Jerk}_{T-\text { mean }}\left(\mathrm{m} / \mathrm{s}^{3}\right)$ & -0.0226 & 0.0664 & -0.0601 & 0.1814 & -1.659 & 1.213 & 0.275 \\
\hline \multirow[t]{6}{*}{ Heavy fog zone } & $V_{F-\text { mean }}(\mathrm{km} / \mathrm{h})$ & 71.154 & 12.593 & 71.715 & 9.578 & -0.008 & 0.040 & 0.842 \\
\hline & $a_{F-\text { mean }}\left(\mathrm{m} / \mathrm{s}^{2}\right)$ & -0.109 & 0.054 & -0.132 & 0.050 & -0.211 & 3.052 & 0.085 \\
\hline & $a_{F-\max }\left(\mathrm{m} / \mathrm{s}^{2}\right)$ & -2.335 & 1.466 & -2.901 & 1.662 & -0.242 & 37.406 & $0.000^{* *}$ \\
\hline & Proportion $_{F-W}$ & 0.351 & 0.080 & 0.377 & 0.079 & -0.007 & 1.745 & 0.191 \\
\hline & Proportion $_{F-T}$ & 0.320 & 0.096 & 0.372 & 0.082 & -0.163 & 5.529 & $0.022^{*}$ \\
\hline & $\operatorname{Jerk}_{F-\text { mean }}\left(\mathrm{m} / \mathrm{s}^{3}\right)$ & -0.0124 & 0.0164 & -0.0117 & 0.0201 & 0.056 & 0.026 & 0.873 \\
\hline
\end{tabular}

Notes: *Significant at the $95 \%$ confidence level; ${ }^{* *}$ significant at the $99 \%$ confidence level. Note Proportion $=\frac{M_{\text {control }}-M_{\text {treat }}}{M_{\text {control }}}$ represent a reduction ratio of the mean indicators between control group and treat group

Figure 5 Indicators of significance

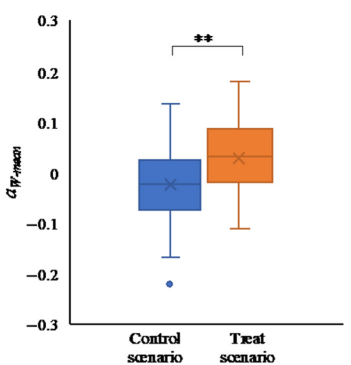

(a)

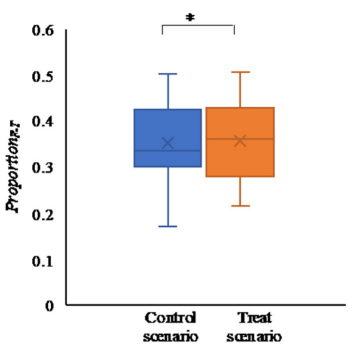

(b)

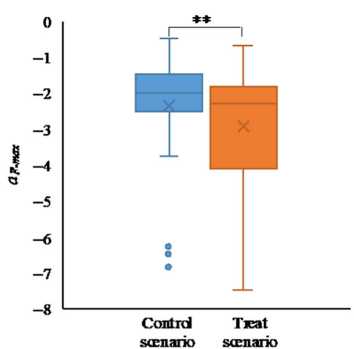

(c)

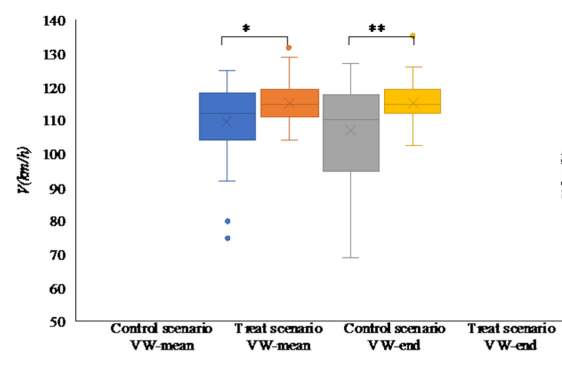

(d)

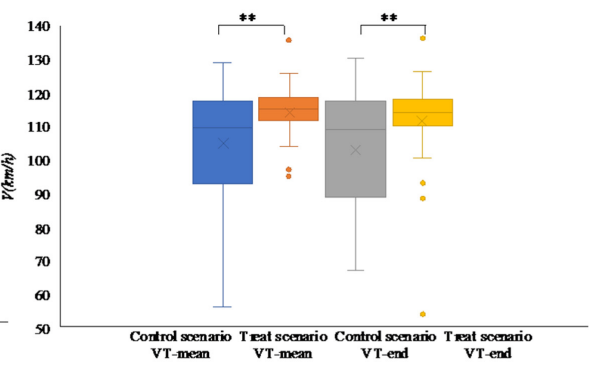

(e)

Notes: (a) $a_{W-m e a n}$; (b) Proportion $F-T$; (c) $a_{F-m a}$; (e) speed in warning zone; (f) speed in transition zone

$$
\begin{aligned}
& \text { Proportion }_{T-W}=\frac{V_{W-\text { mean }}-V_{T-\text { mean }}}{V_{W-\text { mean }}} \\
& \text { Proportion }_{F-W}=\frac{V_{W-\text { mean }}-V_{F-\text { mean }}}{V_{W-\text { mean }}}
\end{aligned}
$$

$$
\text { Proportion }_{F-T}=\frac{V_{T-\text { mean }}-V_{F-\text { mean }}}{V_{T-\text { mean }}}
$$

where $V_{W \text {-mean }}$ is the mean speed in warning zone; $V_{T \text {-mean }}$ is the mean speed in transition zone; $V_{F-\text { mean }}$ is the mean speed in heavy fog zone. It should be noted that sometimes drivers might increase or keep their speed in the fog zone or transition zone. 
In that case, Proportion $_{T-W}$, Proportion ${ }_{F-W}$ and Proportion $_{F-T}$ can be a negative or zero value.

\subsection{Statistical analysis}

\subsubsection{Whole zone}

First, the impact of fog warning system on driving performance in the whole zone needs to be explicated. Therefore, three continuous variables are selected as evaluating indicators, which are $V_{\text {mean }}, a_{\text {mean }}$ ferk mean $_{\text {. }}$.

The trend of overall $V_{\text {mean }}$ is shown in Figure $6(\mathrm{a})$, and spatial significance is shown in Figure 6(b). Because of the influence of foggy weather, the speed generally tends to decline. In the warning zone, the speed change of the treat group is relatively stable and slightly increased. The speed of the control group is slightly decreased at DMS3. As DMS4 indicates that it is the distance of 500 meters away from the heavy fog zone that has a visual impact on the driver, the drivers' speed starts to decrease obviously after DMS4. From the beginning of the transition zone, as the visibility begins to slowly drop, drivers in control group operated relatively stable and continued to reduce the speed. In the case of heavy fog, drivers in treat group continue to slow down. There is little difference between the two scenarios in the heavy fog zone. According to the significance analysis, the velocity changes of the two groups from DMS3 to the end of the gradient region were significantly different.

The trend of overall $a_{\text {mean }}$ is shown in Figure 7(a), spatial significance is shown in Figure $7(\mathrm{~b})$. In the warning zone, drivers in treat group are basically in an accelerated state, but it

\section{Figure 6 Effect of the fog warning system on speed}

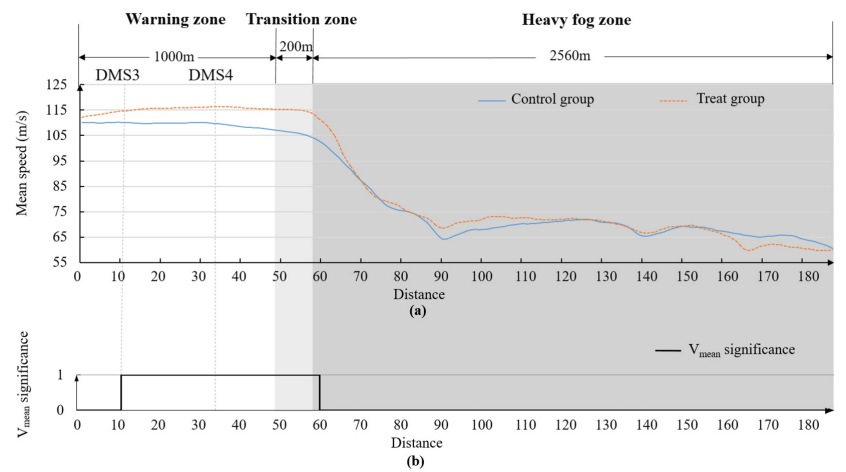

Figure 7 Effect of the fog warning system on acceleration

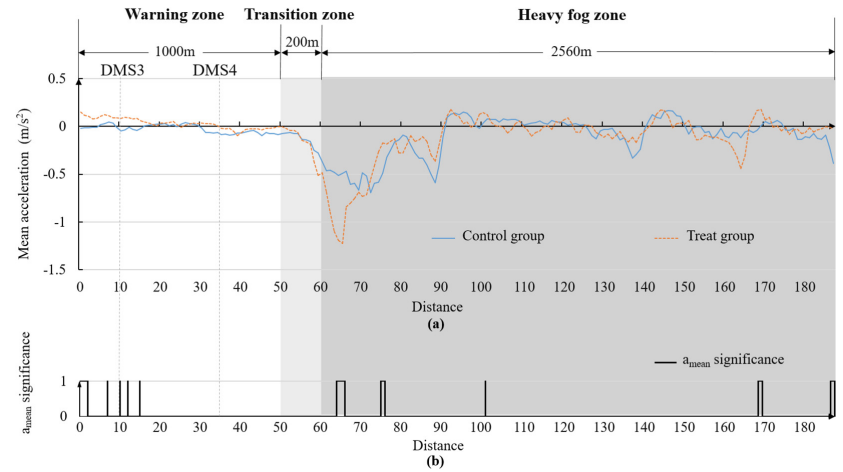

could be seen from the control group that DMS3 has no significant influence on drivers' acceleration. When drivers enter the visual distance of DMS4, the acceleration gradually decreases from positive value to negative value and then continuously decreases with a higher absolute value. In the transition zone, it is obvious that the deceleration of the treat group is greater than that of the control group. At the beginning of the fog zone, it can be concluded that drivers are significantly affected by the visibility, and the reduction speed fluctuation is relatively small as drivers of the control group have received the warning message. However, in the second half of the heavy fog zone, there is no significant difference in acceleration between the two scenarios. According to the significance analysis, there was a significant difference in acceleration between the two groups within the range of DMS3 and at the beginning of the fog zone.

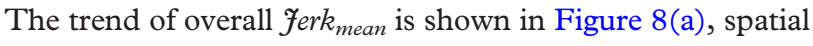
significance is shown in Figure 8 (b). The values of jerk are far away from the axis, which means drivers are more nervous. However, within the visual range of DMS4, the jerk of drivers in control group has a significant decrease, which may be caused by the reason that the warning information make drivers have the slight tension. The values of jerk are not significantly different in the warning zone. In the transition zone, drivers in treat group are more nervous as the visibility drops than those in control group. At the beginning of the fog zone, the tension of drivers increases in both scenarios. Because drivers in control group receive the warning information, it is obvious that the tension of drivers in control group is far less than that those in treat group. In the second half of the heavy fog zone, the tension of drivers in both scenarios decreases without significant difference. According to the significance analysis, jerk was significantly different at DMS3 and at the beginning of fog zone.

\subsubsection{Warning zone}

The corresponding variables in two groups as $V_{W-m e a n}, V_{W-e n d}$ are significant in the warning zone based on a $95 \%$ significance level. $V_{W \text {-mean }}$ represents the mean speed in the warning zone, and $V_{W-e n d}$ indicates the ending speed of the warning zone. As for the corresponding variables in groups, $V_{W-\text { mean }}$ in control group reduces by $5.3 \% ; V_{W-e n d}$ reduces by $7.9 \%$ than that of treat group. The changes of these two indicators indicated that the control group's speed is less than that of treat group in the

Figure 8 Effect of the fog warning system on jerk

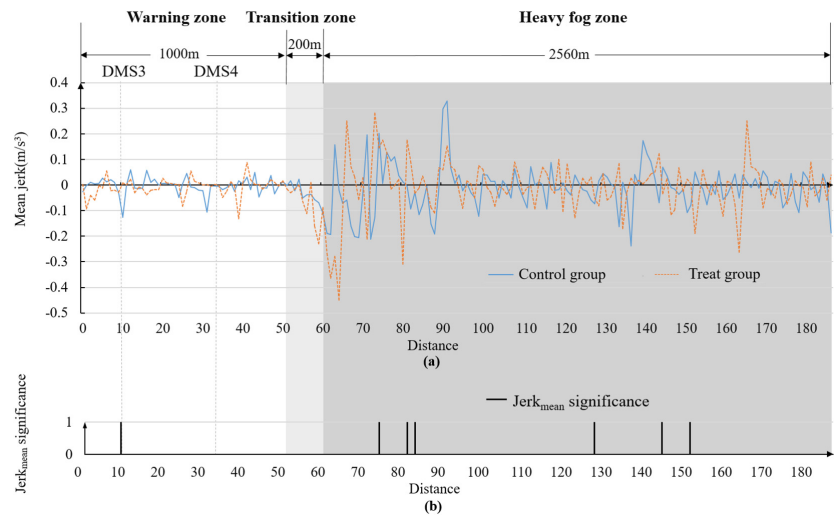


whole warning zone (Table 4, Figure 5). To explore the significant position, the result shows that the $V_{\text {mean }}$ from DMS3 to the end of the warning zone is significant [Figure 6(b)]. Near DMS3, the accelerations of drivers in control group are affected by the warning information and change from the acceleration process to the deceleration process, while the accelerations of drivers in treat group are slowly declined but always in an accelerated state [Figure 7(b)]. At the location of DMS3, the driving tension in control group increases as a result of warning information [Figure $8(\mathrm{~b})$ ]. It indicates that the DMS of the fog warning system can reduce the speed and the acceleration of drivers, and the influence of DMS3 on drivers is greater than DMS4 in warning zone. However, warning messages may make drivers feel slightly nervous.

\subsubsection{Transition zone}

The indicator $V_{T-\text { mean }}$ and $V_{T-\text {-end }}$ are significant based on a $95 \%$ significance level. $V_{T-\text { mean }}$ represents the mean speed in the warning zone, and $V_{W-e n d}$ indicates the ending speed of the warning zone. As for the corresponding variables in groups, $V_{T-\text { mean }}$ in control group reduces by $8.6 \%$ and $V_{W-\text { end }}$ reduces by $8.5 \%$ than that of treat group. The changes of these two indicators indicated that the control group's speed is less than that of treat group in the whole transition zone (Table 4, Figure 5). To explore the significant position, the result shows that the $V_{\text {mean }}$ is significant throughout the transition zone [Figure 6(b)]. It indicates that the fog warning system is beneficial to reduce the speed of entering the fog zone. However, the fog warning system does not reduce the acceleration of entering into the heavy fog zone and ease the drivers' tension.

\subsubsection{Heavy fog zone}

The corresponding variables in two scenarios as $a_{F-\max }$ and Proportion $_{F-T}$ are significant based on a 95\% significance level. $a_{F-\max }$ represents the biggest deceleration in the fog zone, and Proportion $_{F-T}$ represents reduction ratio between the fog zone and transition zone. $a_{F-\max }$ in control group increases by $24.2 \%$ and Proportion P $_{\mathrm{F}}$ reduces by $16.3 \%$ than that of treat group (Table 4, Figure 5). The significant difference of $a_{F-\max }$ and Proportion $_{F-T}$ indicated that in the fog zone, compared with the control group, treat group has the bigger maximum deceleration and the greater deceleration ratio from the transition zone to the fog zone than that of control group. To explore the significant position, the result shows that the mean speed and the mean acceleration of drivers are significant when entering the fog zone, and $\mathcal{F e r k}_{\text {mean }}$ is also significant immediately after entering the heavy fog zone [Figures 6(b), 7 (b) and 8(b)]. However, when drivers adjust to the visibility in the heavy fog zone, the mean acceleration and the mean jerk of drivers show scattered significance because of the limited visual range. It indicates that the fog warning system has a positive effect on speed, acceleration and tension as drivers enter the heavy fog zone. However, the fog warning system has no significant effect on the operation state and the psychological feeling of the whole fog zone.

\section{Discussion}

The purpose of this research is to design a fog warning system for real-time detection of fog weather and build a test platform based on driving simulation technology to evaluate the effectiveness of the fog warning system. To understand the effect of warning system on driving performance better, the experimental road was divided into three zones. The warning zone was to study drivers' driving performance when they observed each DMS. The transition zone was a visual transition created for drivers to adapt to heavy fog better, and can be used to study drivers' responses to changes in visibility. The fog zone was to study the influence of warning information on drivers' driving performance under low visibility conditions. For the above three zones, speed type index, acceleration type index and Jerk were selected to evaluate drivers' driving performance.

First, the speed of drivers was affected by the DMS in the whole zone. Because the drivers in treat group did not received the warning notice ahead of the fog zone and began to slow down continuously in the transition zone. The warning information provided by the DMS is valid, and the warning information that located $1 \mathrm{~km}$ away from the fog area starts to have a greater effect on drivers. Therefore, for the practical application of fog warning system, it can be considered to start the warning at least $1 \mathrm{~km}$ before the fog zone. Second, in this research, the acceleration was used to test driving stability. The early warning concentration and distance of fog to drivers has a positive effect on driving performance. As drivers in control group knew the level of fog in advance and could judge the level of danger, when they felt the reduced visibility, they began to slow down the braking until they reached the ideal speed to drive in the fog area. However, when drivers in treat group felt less visibility, they could not judge the fog level of the final, so they usually took emergency braking. Therefore, it can be concluded that the level of fog should be suggested in the practical application of fog warning system. Finally, in this research, the Jerk was selected to describe the degree of tension of drivers. The reason for drivers' nervousness may be the color of the DMS. Therefore, the font color should be considered in the practical application of fog warning system. It is worth thinking about that the warning information of the fog warning system in this research only mention that makes drivers drive more carefully; However, the research considered that if the speed limit of different fog concentrations is added, the average speed of drivers in the fog zone may be reduced and the safety level can be improved. Therefore, different warning information needs of the DMS need to be further studied in future. This research used the method of spatial significance assessment, which can clearly and accurately obtain the significant difference between all drivers of two different groups at each coordinate point on the whole experimental road. By using this method, the key points and action areas of drivers affected by DMS were obtained in this research, so as to have a more in-depth and accurate understanding of the impact of DMS on drivers.

In addition, driving simulator experiment was conducted in this research, the validity of the driving simulator used in this research has been determined in previous studies (Ding, 2013). The scenario authenticity, brake and speed perception of the simulator have been investigated among more than 300 drivers through subjective questionnaire, and the results show that most drivers agreed with the validity of the simulator. Considering that this research is based on the condition of dense fog which is difficult to control and potentially dangerous in practice, such problems can be evaluated to use driving simulator. 


\section{Conclusion}

Driving in fog zone is potentially dangerous, especially when fog suddenly occurs. The fog warning system can send out the warning information to drivers so that they have psychological expectation of the fog zone to enhance the decision-making of drivers when they are going to enter the fog zone. At present, the DMS of fog warning system only indicates the level of fog. To evaluate the effectiveness of the system, the fog warning system in this research added a distance sensor so that the DMS can indicate the level of fog and the distance from the fog zone. The research established an effective testing platform based on a driving simulator to quantify the effects of fog warning systems by exploring drivers' reactions to the DMS. The concept of graded assessment was used to compare the changes of drivers' decision related to driving performance with or without a fog warning system in multiple indicators in different zones (warning zone, transition zone and heavy fog zone).

The results of the driving simulator experiment are consistent with the actual observation results, which indicates the rationality of the experiment. The results of subjective questionnaire show that drivers accept, understand and trust the fog warning system. The results of the driver performance show that the fog warning system can reduce the speed of drivers when they enter the heavy fog zone, and the speed of drivers in warning zone decreases obviously. However, it has little effect on the speed of the whole heavy fog zone. The fog warning system markedly reduces the acceleration and the tension of drivers while entering the heavy fog zone. Under the influence of the warning information, with the gradual reduction of visibility, the operation stability of drivers at the initial stage is significantly improved, while the impact of the fog warning system on the acceleration of the whole heavy fog zone is not significant. The warning information will make drivers feel slightly nervous. Although the warning information, to some extent, can reduce the tension of drivers when they enter the fog zone at the beginning, it has little effect on the psychology of the whole heavy fog zone.

The fog warning system designed in this research can be used in fog-prone zones to improve safety. The contribution of this research is to provide a comprehensiveness and refinement indicator system for evaluating fog warning system based on a dynamic interactive driving simulation test platform, it is of great significance to the evaluation and application of DMS. At the same time, in the future, this method can provide a general method for the evaluation of vehicle-to-infrastructure technology.

\subsection{Limitations and future research}

The limitation of this paper is that only the driver's performance with or without fog warning system under heavy fog conditions was discussed. Further research is needed to compare the traffic performance with and without the distance information to show the effectiveness of the additional information. At the same time, the reason of the negative comments is worth being explored to help understand how to improve the fog warning system. The subjective questionnaire, visual characteristics and driving behavior changes need to be comprehensively considered to explore the possible negative impact of the warning system, so as to enhance the acceptance of fog warning system in practical application. In addition, a sensitivity analysis on the parameters of the fog warning system needs further exploration, such as the fog density (visibility), the number of DMS, the distance between DMSs or even color and terms used for the warning message. Furthermore, the impact of the traffic volumes and the composition of the volumes on the effectiveness of the fog warning system need to be further concerned. The fog warning system in this research has no significant influence on the speed of the heavy fog zone. To improve the effect of fog warning system on heavy fog area, the control technology based on connected vehicle is expected to be adopted in fog zone.

\section{References}

Abdel-Aty, M., Ekram, A., Huang, H. and Choi, K. (2011), “A study on crashes related to visibility obstruction due to fog and smoke”, Accident Analysis \& Prevention, Vol. 43 No. 5, pp. 1730-1737.

Abdel-Aty, M.A., Oloufa, H., Hassan, M., Ahmed, C., Siddiqui, A.E. and Huang, H. (2010), "Developing an early detection system for reduced visibility", FDOT Report BDK, Vol. 78 No. 5, pp. 62-61.

Brooks, J.O., Crisler, M.C., Klein, N., Goodenough, R., Beeco, R.W., Guirl, C., et al. (2011), "Speed choice and driving performance in simulated fog condition", Accident Analysis E⿱ Prevention, Vol. 43 No. 3, pp. 698-705.

Charlton, S.G. (2009), "Driving while conversing: cell phones that distract and passengers who react", Accident Analysis \& Prevention, Vol. 41 No. 1, pp. 160-173.

Chow, S.C., Shao, J., Wang, H. and Lokhnygina, Y. (2017), Sample Size Calculations in Clinical Research, Chapman and Hall/CRC, FL.

Ding, H., Zhao, X., Rong, J. and Ma, J. (2013), "Experimental research on the effectiveness of speed reduction markings based on driving simulation: a case study", Accident Analysis $\mathcal{E}$ Prevention, Vol. 60 (Complete), pp. 211-218.

Godley, S.T., Triggs, T.J. and Fildes, B.N. (2002), "Driving simulator validation for speed research", Accident Analysis $\mathbb{E}$ Prevention, Vol. 34 No. 5, pp. 589-600.

Hassan, H.M. and Abdel-Aty, M.A. (2011), "Analysis of drivers' behavior under reduced visibility conditions using a structural equation modeling approach", Transportation Research Part F: traffic Psychology and Behaviour, Vol. 14 No. 6, pp. 614-625.

Hawkins, R.K. (1988), "Motorway traffic behaviour in reduced visibility conditions", Vision in Vehicles II. Second International Conference on Vision in Vehicles Applied Vision Association Ergonomics Society Association of Optometrists, pp. 9-18.

Hoogendoorn, R.G., Tamminga, G., Hoogendoorn, S.P. and Daamen, W. (2010), "Longitudinal driving behavior under adverse weather conditions: adaptation effects, model performance and freeway capacity in case of fog", 13th International IEEE Conference on Intelligent Transportation Systems, Funchal, Portugal, 2010, pp. 450-455, doi: 10.1109/ ITSC.2010.5625046.

Lerman, J. (1996), "Study design in clinical research: sample size estimation and power analysis", Canadian fournal of Anaesthesia, Vol. 43 No. 2, pp. 184-191. 
Pilié, R.J., Mack, E.J., Kocmond, W.C., Rogers, C.W. and Eadie, W.J. (1975), "The life cycle of valley fog. part I: micrometeorological characteristics", fournal of Applied Meteorology, Vol. 14 No. 3, pp. 347-363.

Snowden, R.J., Stimpson, N. and Ruddle, R.A. (1998), "Speed perception fogs up as visibility drops", Nature, Vol. 392 No. 6675, p. 450.

Vanderhaegen, F., Zieba, S., Enjalbert, S. and Polet, P. (2011), "A benefit/cost/deficit (BCD) model for learning from human errors", Reliability Engineering \& System Safety, Vol. 96 No. 7, pp. 757-766.

Wu, Y., Abdel-Aty, M., Park, J. and Selby, R.M. (2018), "Effects of real-time warning systems on driving under fog conditions using an empirically supported speed choice modeling framework", Transportation Research Part C: emerging Technologies, Vol. 86, pp. 97-110.

Zaki, M.H., Sayed, T. and Shaaban, K. (2014), "Use of drivers' jerk profiles in computer vision-based traffic safety evaluations", Transportation Research Record: Fournal of the Transportation Research Board, Vol. 2434 No. 1, pp. 103-112.

Zhao, X., Xu, W., Ma, J., Li, H., Chen, Y. and Rong, J. (2019), "Effects of connected vehicle-based variable speed limit under different foggy conditions based on simulated driving", Accident Analysis \& Prevention, Vol. 128, pp. 206-216.

\section{Further reading}

Al-Ghamdi, A.S. (2007), "Experimental evaluation of fog warning system", Accident Analysis \& Prevention, Vol. 39 No. 6, pp. 1065-1072.

Boyle, L.N. and Mannering, F. (2004), "Impact of traveler advisory systems on driving speed: some new evidence", Transportation Research Part C: Emerging Technologies, Vol. 12 No. 1, pp. 57-72.
Chun-Hao, Z. (2008), Statistical Analysis and Research on Motorway Cycling Accidents, Chang' an university.

Goodwin, L.C. and Pisano, P. (2003), Best Practices for Road Weather Management, Road Weather.

Grade of fog forecast (2012), "gbt 27964-2011", (accessed 1 March).

Juan, W. (2009), Research on Releasing Content and Setting Position of Variable Information Signs on Freeway, Chang' an university.

Pisano, P.A. and Goodwin, L.C. (2004), "Research needs for weather-responsive traffic management", Transportation Research Record: Fournal of the Transportation Research Board, Vol. 1867 No. 1, pp. 127-131.

Specification for Layout of Highway Traffic Signs and Markings (2009), “jtg d82-2009”, (accessed 1 October).

Van Der Horst, R. and De Ridder, S. (2018), "Influence of roadside infrastructure on driving behavior: driving simulator study", Transportation Research Record: Fournal of the Transportation Research Board, pp. 36-44.

Wei-Han, Z. (2009), Traffic Safety Guarantee Technology in Foggy Area of Freeway, China Communicationa Press.

Xian-Feng, X. (2007), Research on Highway Safety Control under Adverse Weather Conditions, Shandong university of science and technology.

Xue-Hao, C. (2010), Analysis of Highway Traffic Accidents Causes and Traffic Control Points in the Fog, China Public Security.

Zhao, X. and Sarasua, W.A. (2018), "How to use driving simulators properly: impacts of human sensory and perceptual capabilities on visual fidelity", Transportation Research Part C: Emerging Technologies, Vol. 93, pp. 381-395.

Zhen-Ye, W., Jun, C., Wan-Xiu, L. and Song, G. (2016), "Analysis on the logic and real vehicle test of the body stability control system", Automotive Practical Technology, No. 6, pp. 136-139.

\section{Corresponding author}

Haijian Li can be contacted at: lihaijian@bjut.edu.cn 